\title{
Atmospheric Interaction with Nanosatellites from Observed Orbital Decay
}

DOI:

10.1016/j.asr.2018.02.022

Document Version

Accepted author manuscript

Link to publication record in Manchester Research Explorer

\section{Citation for published version (APA):}

Macario Rojas, A., Smith, K., Crisp, N., \& Roberts, P. (2018). Atmospheric Interaction with Nanosatellites from Observed Orbital Decay. Advances in Space Research, 61(12), 2972-2982.

https://doi.org/10.1016/j.asr.2018.02.022

\section{Published in:}

Advances in Space Research

\section{Citing this paper}

Please note that where the full-text provided on Manchester Research Explorer is the Author Accepted Manuscript or Proof version this may differ from the final Published version. If citing, it is advised that you check and use the publisher's definitive version.

\section{General rights}

Copyright and moral rights for the publications made accessible in the Research Explorer are retained by the authors and/or other copyright owners and it is a condition of accessing publications that users recognise and abide by the legal requirements associated with these rights.

\section{Takedown policy}

If you believe that this document breaches copyright please refer to the University of Manchester's Takedown Procedures [http://man.ac.uk/04Y6Bo] or contact uml.scholarlycommunications@manchester.ac.uk providing relevant details, so we can investigate your claim.

\section{OPEN ACCESS}




\title{
Atmospheric Interaction with Nanosatellites from Observed Orbital Decay
}

\author{
A. Macario-Rojas*, K. L. Smith, N. H. Crisp, P. C. E. Roberts \\ School of Mechanical Aerospace and Civil Engineering \\ The University of Manchester \\ M13 9PL, United Kingdom
}

\begin{abstract}
Nanosatellites have gained considerable presence in low Earth orbits wherein the atmospheric interaction with exposed surfaces plays a fundamental role in the evolution of motion. These aspects become relevant with the increasing applicability of nanosatellites to a broader range of missions objectives. This investigation sets out to determine distinctive drag coefficient development and attributes of atmospheric gas-surface interactions in nanosatellites in the common form of standard 3U CubeSats from observed orbital decay. As orbital decay can be measured with relative accuracy, and its mechanism broken down into its constituent sources, the value of drag-related coefficients can be inferred by fitting modelled orbit predictions to observed data wherein the coefficient of interest is the adjusted parameter. The analysis uses the data of ten historical missions with documented passive attitude stabilisation strategies to reduce uncertainties. Findings indicate that it is possible to estimate fitted drag coefficients in CubeSats with physical representativeness. Assessment of atomic oxygen surface coverage derived from the fitted drag coefficients is broadly consistent with theoretical trends. The proposed methodology opens the possibility to assess atmospheric interaction characteristics by using the unprecedented opportunity arising from the numerous observed orbital decay of nanosatellites.
\end{abstract}

Keywords: nanosatellite, drag coefficient, orbit decay, atomic oxygen surface coverage

\section{Introduction}

Typical operative altitudes for nanosatellites lie within one thousand kilometres in orbits wherein the dominant nonconservative disturbing force is derived from atmospherespacecraft interaction. This interaction causes monotonic reduction of orbital eccentricity and semi-major axis, and can produce attitude perturbation torques. These effects are in the main undesirable during the operational life of nanosatellites due to characteristic low mass and volume efficiencies that hinder the establishment of flexible system margins. In the framework of drag force theory, the drag coefficient $\left(C_{D}\right)$ captures the intricacies of atmosphere-surface interactions such as energy and momentum interchange mechanisms. Amongst the drag force constituents, $C_{D}$ is normally the least tightly defined quantity, which is also subject to significant variation according to atmospheric characteristics. Existing in-situ measurements in Very Low Earth Orbits (VLEO) ( $\sim 150-300 \mathrm{~km}[15]$ ) have shown that atomic oxygen may populate exposed surfaces greatly controlling interactions' quality and therefore $C_{D}$. Acknowledging the key importance of atmospheric drag to the mission and application diversification of nanosatellite platforms, the investigation presented herein sets out to estimate atomic oxygen surface coverage in nanosatellites from observed orbital decay.

\footnotetext{
* Corresponding author

Email addresses: alejandromacario.rojas@manchester .ac .uk (A.

Macario-Rojas), kate.smith@manchester.ac.uk (K. L. Smith),

nicholas.crisp@manchester.ac.uk (N. H. Crisp),

peter.c.e.roberts@manchester.ac.uk (P. C. E. Roberts)
}

The proposed method involves a deductive process in which the macroscopic atmospheric interaction characterising the natural evolution of an orbit time span, is captured in fitted values of $C_{D}$, hereafter referred to as $C_{D F}$. Finally, the atomic oxygen surface coverage is estimated from $C_{D F}$ and the theoretical weighted contributions of extreme $C_{D}$, i.e. those of a clean and fully contaminated surface.

Atmospheric interaction with exposed surfaces can be explained in terms of the kinetic theory of gases. Gas-surface interaction phenomena or GSIs, in particular those related to the interchange of momentum and energy with a surface, are relevant for this investigation. Under the prevailing atmospheric conditions at LEO altitudes, molecules travel large distances compared to a characteristic length before intermolecular collisions can occur [26]. In addition, particles may manifest bulk ordered and random translational motion. With respect to a reference surface immersed in an atmosphere showing prevalently ordered motion, the stream would move with a distinctive relative speed $v_{r}$. To account for the fraction of particles in random translational motion, a Maxwellian velocity distribution (thermal velocity) is typically assumed. The bulk and thermal speed contributions of the particles are related in the dimensionless molecular speed ratio $s$ defined by Eq. (1), wherein $k_{B}$ is the Boltzmann constant, $T_{\infty}$ the freestream temperature, and $\bar{m}_{m}$ the mean molecular mass.

$$
s=v_{r} \sqrt{\frac{\bar{m}_{m}}{2 k_{B} T_{\infty}}}
$$


From this relationship note that if the value of $s$ is large then the bulk motion is dominant, whilst a small value would mean significant randomness in the motion of the particles. For values of $s>5$, the bulk atmospheric motion is sufficiently predominant to assume a collimated stream of particles [6], in the so called hyperthermal approximation. Values of $s>5$ are normally found at orbital altitudes below $\sim 600 \mathrm{~km}$ where heavy atmospheric species and ordered bulk motion prevail. On the other hand, random translational motion is dominant at high altitudes where light species in a rarefied atmosphere are easily energised by environmental radiation. GSIs are complex

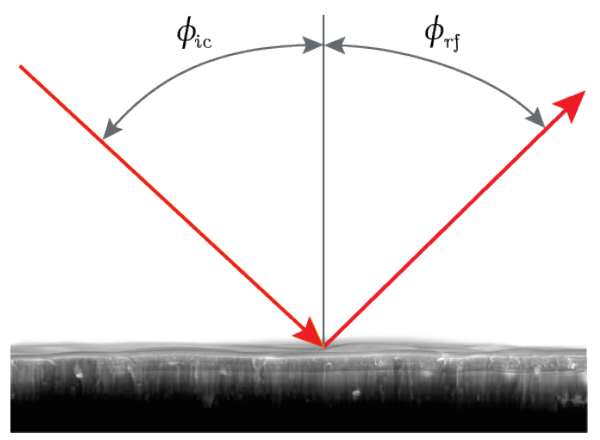

(a) Specular reflection originated by a clean surface.

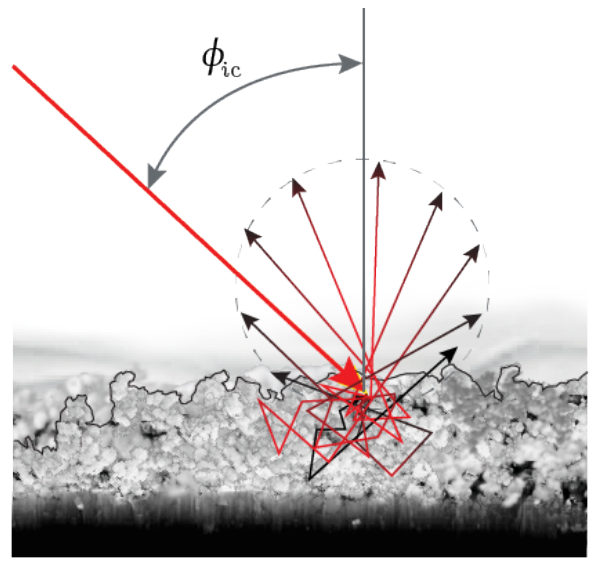

(b) Diffuse reflection chiefly originated by the presence of adsorbate and reaction products.

Figure 1: Angular distributions of scattered particles.

processes that involve to some extent chemical reactions, mass transfer, and momentum and energy exchange. The force exerted by the molecules over the interaction surface through collisions depends on the way the particle impingement occurs. This interaction is greatly dependent on the surface roughness relative to the size of the impinging particles [25], the quality of which is manifested by the resulting particle reflection. Assuming that the incident particles behave like hard spheres, a rough surface would cause several internal reflections before the particles find a way out through the interstices of the surface. Along each of several internal reflections, as shown in Fig. $1 b$ the particles deliver part of their kinetic energy and receive thermal energy from the surface. Under these circumstances the overall energy exchange process may result in unre- lated incidence and re-emission directions. The collective effect of incident particles on a rough material is thus a characteristic diffuse reflection pattern as the bulk incoming direction information is gradually lost. On the other hand, for a hypothetical surface allowing only a single reflection, Fig. 1a, the outgoing particles follow a specular pattern with the same angle of incidence, i.e. $\phi_{r f}=\phi_{i c}$, but not necessarily the same speeds as the exchange of energy still exists. Complementarily, intermediate cases are possible for a spectrum of surface conditions resulting in a quasi-specular reflection. In principle, the force delivered during a GSI can be fully described by momentum transfer. The minimum number of independent components required to parametrise the momentum transfer is two, the so-called normal and tangential momentum accommodation coefficients $\sigma_{n}$ and $\sigma_{t}$ respectively. However, as the energy accommodation coefficient $\alpha$ is traditionally used in the analysis of gas-surface heat transfer in general physics and because in practice energy transfer is often easier to measure than momentum, it is common to find in literature GSI force models using a mix of momentum and energy accommodation coefficients.

A GSI can be characterised by the fractional energy accommodation coefficient $\alpha$ in Eq. (2). It is used to express the level of equilibrium attained by the kinetic energy of the incident particles with respect to the thermal energy of the surface before re-emission. If the particles are re-emitted in thermal equilibrium with the surface, $E_{r f} \equiv E_{w}$, then $\alpha$ is unity. Conversely, if no energy exchange exists, $E_{i c} \equiv E_{r f}, \alpha$ is equal to zero. At this point is important to emphasise the unambiguity of the total energy exchange during the GSI and the energy accommodation coefficient concepts. Actual impinging particles have different degrees of freedom, i.e. translation, rotation and vibration, at the moment of the interaction. Degrees of freedom are particularly relevant when the impinging particle is effectively asymmetric. In that case, it is convenient to introduce separate energy accommodation factors for each degree of freedom [25, 26]. However, for the purpose of this investigation, the impinging particles are assumed symmetric.

$$
\alpha=\frac{E_{i c}-E_{r f}}{E_{i c}-E_{w}}
$$

The normal and tangential momentum accommodation coefficients are used to parametrise the momentum transfer in a GSI. The tangential momentum accommodation coefficient in Eq. (3) is derived from a general form formula similar to Eq. (2) with null surface momentum $\left(\tau_{w}=0\right)$. In order to describe the variables in $\sigma_{t}$ we need to turn our attention to the angular distribution of scattered particles in Fig. 1. In the ideal case of specular reflection, the incident tangential momentum of a particle, $\tau_{i c}$, has to be equal to the reflected tangential momentum $\tau_{r f}$ that is $\sigma_{t}=0$. On the other hand, if the surface condition prompts the complete loss of information about the incoming direction of the particle, the axis of the lobe demarcating the possible directions after re-emission is centred in the normal surface vector, or $\tau_{r f}=0$. In such a case $\sigma_{t}=1$. It is interesting to note that if the particle is backscattered $\tau_{r f}<0$ and $\sigma_{t}$ can be greater than unity. However, the analysis of $\sigma_{t}$ is usually restricted to the interval $[0-1]$. Empirical evidence shows that 
$\sigma$ and $\alpha$ are positively linked to the mass of the incident particles and the level of surface contamination. On this point, it has been observed in Earth orbiting objects that the atomic oxygen present in the atmosphere greatly interacts with exposed surfaces enhancing energy accommodation and diffuse reflection [16].

$$
\sigma_{t}=1-\frac{\tau_{r f}}{\tau_{i c}}
$$

The atmospheric composition varies with altitude. It is expected that through various physical phenomena, e.g. chemical affinity, some atmospheric gas species populate exposed surfaces. In particular, the presence and interaction of atmospheric atomic oxygen $(\mathrm{AO})$ on spacecraft materials has been identified through in-orbit mass spectrometry measurements [19], and from the analysis of retrieved materials exposed to the space environment in near LEO orbits [7]. High-energy incoming solar radiation, mainly in the form of EUV and X-ray, causes atomic oxygen to become relatively abundant at $\sim 100 \mathrm{~km}$ where its number density peaks derived from dissociation of oxygen allotropes. Depending on the incoming solar radiation, the relative abundance of $\mathrm{AO}$ over other atmospheric species may reach altitudes as high as $700 \mathrm{~km}$ normally populated by lighter species i.e. He and $\mathrm{H}$. Atomic oxygen is highly reactive and binds strongly to almost every satellite material causing erosion and oxidation.

During the 1960s, at the dawn of the space age, various values of drag coefficients were in common use. The employment of the range $2.0-2.3$, mainly 2.2 , was justified by laboratory estimations on clean surfaces [6, 11, 40] and by knowledge gaps about GSI in real space environment conditions. At the present time it is believed that spacecraft surface material properties are likely to be masked by atomic oxygen, even at altitudes of $\backsim 700-800 \mathrm{~km} \mathrm{[22,} \mathrm{14],} \mathrm{controlling} \mathrm{the} \mathrm{type} \mathrm{of} \mathrm{reflec-}$ tion and range of energy accommodation coefficient. Hence, the value of $C_{D}$ is a strong function of $\mathrm{AO}$ abundance. With the use of the kinetic theory of gases and available empirical data, GSI models have been developed to enable closed-form solutions for the estimation of physical drag coefficients. At this point it is important to emphasise that free molecular closedform solutions generally support single re-emission of particles only, like those occurring on convex geometries as illustrated in Fig. 2. Conversely, if the satellite geometry is concave, then remitted particles are likely to undergo subsequent reflections making the use of dedicated numerical analysis necessary. This investigation uses the Schaaf and Chambre (SC) closed solution for the drag coefficient of a flat plate in free molecular flow (Maxwellian flow) [33]. The SC closed form solution is a function of $\sigma_{n}, \sigma_{t}, s$, the average normal speed of diffusely re-emmited particles $V_{w}$, the relative speed of the incident particles $V$, and the flat plate angle of attack 1 . In order to transform the SC closed form solution in terms of $\alpha$ for the purposes of this investigation, we use the Cercignani-Lampis-Lord GSI model modifications reported in the work of Walker et al. [39]

\footnotetext{
${ }^{1}$ The relationship between the angle of attack and bulk angle of incidence (assuming hyperthermal approximation) is given by $\cos \phi_{i c}=\sin B$.
}

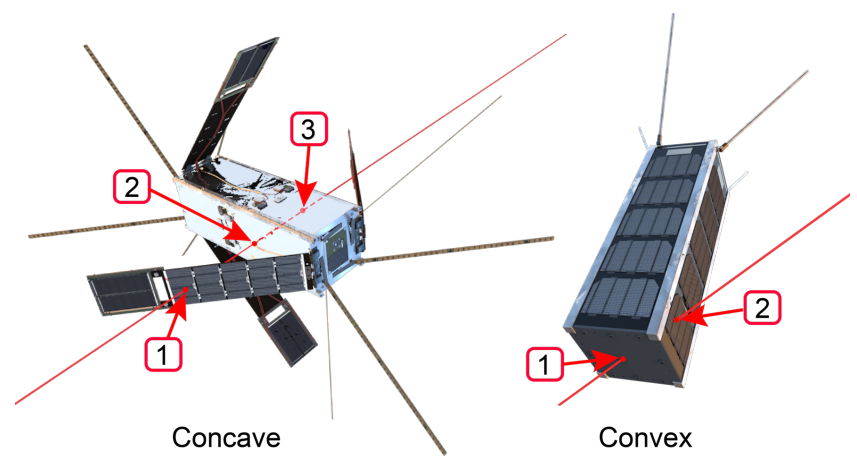

Figure 2: Identification of concave and convex nanosatellite geometries. In convex geometries an imaginary straight line passing through the nanosatellite body in any direction crosses the external surfaces only two times, otherwise the geometry is concave.

yielding Eq. (4) where $C_{I, j}, C_{I I, j}$, and $C_{I I I, j}$ are given by Eq. (5), Eq. (6), and Eq. (7) respectively. The best-fit parameters, $\beta, \gamma$, $\delta$, and $\zeta$, are presented in Table 1 . With these modifications the SC closed form solution is expressed in terms of $\alpha_{n}$ (part of the kinetic energy due to normal motion [5] but representative of the total $\alpha$ ) instead of $\sigma_{n}$, and the surface temperature $T_{s}$ and atmospheric translational temperature $T_{\infty}$ instead of $V_{w}$ and $V$. A final remark about this drag coefficient equation is that all portions of the flat plate experience impingement due to the random motion of the molecules (rear and front sides). The total physical drag coefficient $C_{D}$ for a mixture of $N$ number of gases is given by the weighted sum of the individual contribution of the atmospheric species $\left(C_{D, j}\right)$ in Eq. (8), with $\chi_{j}$ as their respective mole fraction [22, 39].

$$
\begin{gathered}
C_{D, j}=C_{I, j}+C_{I I, j}+C_{I I I, j} \\
C_{I, j}=2\left[\sigma_{t} \cos ^{2} B+\left(2-\sqrt{1-\alpha_{n}}\right)\left(\frac{1}{2 s^{2}}+\sin ^{2} B\right)\right] \times \\
\\
\sin B \operatorname{erf}(s \sin B) \\
C_{I I, j}=\frac{2}{\sqrt{\pi} s}\left[\sigma_{t} \cos ^{2} B+\left(2-\sqrt{1-\alpha_{n}}\right) \sin ^{2} B\right] e^{-s^{2} \sin ^{2} B} \\
C_{I I I, j}=\zeta_{j} e^{-\beta_{j}\left(1-\alpha_{n}\right)^{\gamma_{j}}}\left(\frac{T_{s}}{T_{\infty}}\right)^{\delta_{j}+\frac{1}{2}} \frac{\sqrt{\pi}}{s^{2}} \sin ^{2} B \\
C_{D}=\frac{1}{\bar{m}_{m}} \sum_{j=1}^{N} \chi_{j} m_{j} C_{D, j}
\end{gathered}
$$

Nonconservative perturbation forces acting on satellites, e.g. atmospheric drag and solar-radiation pressure, depend strongly on the state and disposition of surfaces. The angle of interaction between the perturbing vector and surface elements greatly defines the collective reaction vector on the spacecraft. Therefore, high fidelity perturbation estimations must account for the joint effect of various surface relative dispositions and materials. In practice, simplified geometric abstractions and material combinations are normally used to achieve tailored levels of exact- 
Table 1: Best-fit parameters for a flat plate [39]

\begin{tabular}{lrrrrc}
\hline Species & $\boldsymbol{\beta}$ & $\boldsymbol{\gamma}$ & $\boldsymbol{\delta}$ & $\boldsymbol{\zeta}$ & $\begin{array}{c}\text { Atomic } \\
\text { mass }\end{array}$ \\
\hline $\mathrm{O}_{2}$ & 6.300 & 0.260 & 0.420 & 20.500 & 32 \\
$\mathrm{~N}_{2}$ & 6.600 & 0.220 & 0.480 & 35.000 & 28 \\
$\mathrm{AO}$ & 5.850 & 0.200 & 0.480 & 31.000 & 16 \\
$\mathrm{~N}$ & 4.900 & 0.320 & 0.420 & 8.000 & 14 \\
$\mathrm{He}\left(0.95<\alpha_{n}<1.00\right)$ & 6.200 & 0.380 & 3.300 & 0.740 & 4 \\
$\mathrm{He}\left(0.90<\alpha_{n}<0.95\right)$ & 3.800 & 0.520 & 3.400 & 1.120 & 4 \\
$\mathrm{He}\left(0.50<\alpha_{n}<0.90\right)$ & 3.450 & 0.520 & 2.400 & 0.930 & 4 \\
$\mathrm{He}\left(0.00<\alpha_{n}<0.50\right)$ & 0.080 & 0.520 & 4.200 & 1.100 & 4 \\
$\mathrm{H}\left(0.95<\alpha_{n}<1.00\right)$ & 3.900 & 0.195 & 1.400 & 0.300 & 1 \\
$\mathrm{H}\left(0.90<\alpha_{n}<0.95\right)$ & 3.500 & 0.420 & 2.000 & 0.720 & 1 \\
$\mathrm{H}\left(0.50<\alpha_{n}<0.90\right)$ & 3.450 & 0.520 & 2.400 & 0.930 & 1 \\
$\mathrm{H}\left(0.00<\alpha_{n}<0.50\right)$ & 0.095 & 0.465 & 2.900 & 0.920 & 1 \\
\hline
\end{tabular}

ness [10]. In most nanosatellite missions it is infeasible or impractical to keep a continuous track of attitude to assess precise long term perturbation effects. Rather, characteristic invariable cross-sectional areas $\left(A_{s t a}\right)$ are commonly used in rough estimations. For example, some studies have reported results using the statistical surface area value from multiple projected areas with respect to all possible view angles [18]. This approach may represent a practical solution to perturbation quantification from unknown satellite attitude. However, aiming at the maximum possible quality from the fitting process presented in this investigation, efforts are directed towards the reduction of the principal sources of uncertainty such as cross-sectional areas. In order to tackle the level of uncertainty of invariable crosssectional areas, this investigation implements dynamic crosssectional areas $\left(A_{d y n}\right)$ able to capture a higher level of detail along the attitude and orbital motion evolution. To this purpose, 3D computational models of the satellites and the ability to effectively estimate attitude dynamics are indispensable. In spite of the lack of long-term continuous attitude tracking, as mentioned before, it is still possible to evaluate attitude dynamics in nanosatellites furnished with effective passive attitude control systems. The premiss for this possibility, is that passive attitude control systems are normally dependent upon an wellestablished external stabilising vector. This topic is discussed in detail in Section 2 .

The observational orbital data used in the fitting process in this investigation, is provided by two-line element sets (TLE) from the US Strategic Command (USSTRATCOM) open catalogue [30]. The TLE set is a technical tool used to convey condensed orbital information from observations to specialised orbit propagator software. In general terms, the information coded in the TLE sets encloses observed mean orbital elements using the SGP4 orbital model [36, 12]. Precise ephemeris knowledge is unattainable with the use of TLE sets as errors could be of more than $1 \mathrm{~km}$ due to acquisition method margins, the Kozai mean values used for the semi-major axis and mean motion derivation, and forced rounded values required for publication [37]. Despite the inherent coarse attributes of the TLE sets, these still support a variety of detailed technical analy- ses [13, 24, 19]. The BSTAR drag term $\left(B^{*}\right)$ reported in the TLE sets, is a differential free correction factor used to fit the SGP4 orbital model to observations. The $B^{*}$ may not be related predominantly to atmospheric drag since the correction may be due to solar-radiation pressure, third-body perturbations, or mismodeled factors. However in atmospheric drag dominated orbits, $B^{*}$ may provide rough information about the ballistic coefficient $B C$ [36] allowing the estimation of $C_{D}$ according to the aerodynamic theory, i.e. $B C=m /\left(C_{D} A\right)$. On the other hand, the fitting method for drag coefficient estimation aims to infer the level of drag by adjusting iteratively a high fidelity orbital model comprised of precise perturbation models, to the observed orbit evolution profile reported by the TLE sets. The fundamental difference between $C_{D}$ estimations from the fitting method and estimations from the $B^{*}$, is that those from a fitting method incorporate information of the space environment to weight perturbation contributions, thus filtering information about drag coefficients with more efficiency and reliability.

\section{Simulation and Analysis}

For most satellites in near LEO orbits, the drag force contribution to the orbital evolution in orbital parameters is high enough to be clearly distinguished amongst other perturbations. The successful identification of perturbation components and their contributions is possible by virtue of precise space environment and space-spacecraft interaction models. In this manner, the resolution of the recognised contributions relies largely on the quality of the models. If the models are precise and are able to reproduce spatial and temporal changes of the perturbations, then it is feasible to propagate orbits with high fidelity. In principle, an ideal observed initial orbital state can be propagated over a period of time using a high precision orbit propagator to match a subsequent second ideal observed state obtaining equivalent results. However in practice many real factors alter the equivalence of observed and propagated data. Assuming that the initial and final observed orbital states are precise, judicious adjustments of model variables prone to fluctuations may improve propagations disclosing the magnitude of their real variations. In drag dominated orbits, the value of the drag coefficient is the least tightly defined variable subject to fluctuation. In such a scenario the fitted drag coefficient would enable analogous observed and propagated orbital states. The selection of missions for this investigation are based upon open availability of data, reported passive attitude control schemes, and predominant convex geometry in $3 \mathrm{U}$ CubeSats. The motivations behind the selection of the $3 \mathrm{U}$ size factor are the varied number of available missions covering a rich range of space environment conditions. Table 2 presents the selected CubeSat missions. From available information nine CubeSat missions fulfilling the investigation criteria were identified. In addition to these missions and profiting from geometric adequacy and data availability, the BEVO 2 CubeSat is incorporated taking into consideration that it was apparently not activated before deployment and presumably its active attitude control system is inoperable. Therefore in this investigation BEVO 2 is assumed 
Table 2: List of selected 3U CubeSats

\begin{tabular}{|c|c|c|c|}
\hline Name & Attitude Control & Mass [kg] & Launch \\
\hline BEVO 2 & None * & 5.0 & $12 / 2015$ \\
\hline ExoCube $^{\dagger}$ & $\nabla G$ & 4.0 & $01 / 2015$ \\
\hline GEARRS 1 & PMAC & 3.9 & $07 / 2014$ \\
\hline GeneSat- $1^{\dagger}$ & PMAC & 4.1 & $12 / 2006$ \\
\hline PharmaSat $1^{\dagger}$ & PMAC & 4.5 & $05 / 2009$ \\
\hline RAX-2 & PMAC & 2.8 & $10 / 2011$ \\
\hline SMDC-ONE 1 & PMAC & 4.0 & $12 / 2010$ \\
\hline SporeSat $1^{\dagger}$ & PMAC & 5.5 & $04 / 2014$ \\
\hline $\mathrm{S}^{\mathrm{C} C U B E^{\dagger}}$ & $\nabla G$ & 3.9 & $08 / 2015$ \\
\hline TurkSat-3USat & PMAC & 4.0 & $04 / 2013$ \\
\hline
\end{tabular}

* Assumed from failed systems activation

$\dagger$ Approximated standard geometry

to be tumbling in an uncontrolled manner. Fig. 3 shows the orbital evolution of the selected CubeSats alongside the sunspot number with the purpose of representing general fluctuations in the space environment. From the figure the varied range of orbital altitudes and space weather conditions analysed in this investigation are observed.

The attitude control schemes for each selected mission are provided in Table 2 It is important to bear in mind that the selection of these attitude control schemes is based on the fundamental importance of establishing $A_{d y n}$ for estimation of perturbations, i.e. drag and SRP. The instantaneous $A_{d y n}$ is estimated with the use of a simple three-dimensional computational model of a 3U CubeSat (cuboid) at the expected attitude, and the relevant perturbing reference vector. Reported steady state attitude behaviour controlled by Passive Magnetic Attitude Control (PMAC) systems shows coarse magnetic lock to the local Earth magnetic vector, with worst-case dipole alignment of less than $\backsim 20^{\circ}$ in RAX-2 [32]. For the sake of analysis homogeneity, this investigation assumes in all PMAC cases, complete alignment of the PMAC dipole to the local Earth magnetic field modelled with the Wold Magnetic Model [2]. In the case of gravity-gradient $(\nabla G)$ stabilisation, the CubeSats' axis of minimum moment of inertia is aligned to the local gravitational acceleration vector computed with the GRACE+GOCE Gravity Model GGM05G [1] using harmonics up to 240. As in the PMAC case, perfect alignment is assumed in all instances.

A central element in atmospheric drag analysis is the performance of the atmospheric density model in reproducing actual attributes. The fidelity of the atmospheric density model is fundamental in this investigation because it largely establishes orbital decay estimations and therefore the precision of fitted drag coefficients. This investigation uses the Jacchia-Bowman Thermospheric density model 2008 (JB2008) [3]

\footnotetext{
${ }^{2}$ It is worthwhile to mention that an atmospheric model can hardly fulfil all applications performance. However, the selection of the JB2008 is based on the recommendation of the ECSS [9] regarding the use of using JB2006 for atmospheric density calculations above $120 \mathrm{~km}$, The CIRA Working Group [34] ratification of the JB2008 for preferred use above the same orbital altitude, and the JB2008 performance tests reported by Shim et al. [27], Vaughan [38], where JB2008 is more often better than other atmospheric models for this altitude range.
}

To exemplify an important repercussion of the atmospheric density model fidelity on fitted values, notice that overestimated values of $\bar{m}_{m}$ cause proportional increments in $s$ for fixed $T_{\infty}$ and $v_{r}$ (Eq. (1)). This in turn reduces $C_{D}$ chiefly due to $C_{D} \propto s^{-2}$. It is thus reasonable to compensate the overestimation in the atmospheric density by increasing the $C_{D}$ value with the corresponding identified bias percentage [19, 20]. In this respect, Pardini et al. [20] identified density bias in an earlier version of the JB2008 thermospheric model during the $23^{\text {rd }}$ sunspot maximum ${ }^{3}$ However the current version of the JB2008, used in this investigation, has been subjected to index corrections [29]. This fact and the conditions of the ongoing $24^{\text {th }}$ sunspot cycle make necessary updated studies to identify bias in the current version of the JB2008 model.

The fitting algorithm implemented in this investigation can be divided into two main operations. Firstly, the data provided in the assembly of TLE sets require proper interface from its native SGP4 form to the bespoke special perturbation technique Orbit Propagator $(\mathrm{OP})^{4}$ designed for the study. To this end the retrieved mean state vector at epoch from a TLE set is converted from its native True Equator Mean Equinox (TEME [12, 36]) coordinate system to the True equator Of the Date (TOD) reference system required by the OP. Subsequently, the orbital propagation using a seed value of $C_{D_{i}}$ is carried out as follows. A propagated orbit $O P_{t_{N-1}}$ in Fig. 4 from initial orbital data $T L E_{N-1}$, is terminated by the availability of a subsequent observed data $T L E_{N}$. Thus, the new epoch time $t_{N}$ defines $O P_{t_{N i}}$. The residual value on SMA between the $i$-th propagation and $N$ th observation at same epoch, $R_{S M A_{i}}$, is then saved for analysis. In this regard each pair of TLE sets, i.e. $T L E_{N-1}$ and $T L E_{N}$, define irregular propagation periods that together with accuracy and resolution concerns imposed by the TLE-OP-TLE assessment may entail unrepresentative $R_{S M A_{i}}$ values. Consequently this investigation bounds the analysis to plausible orbital decay values, explained below, as a mitigating measure.

The second main operation is the fitting of $C_{D}$, through the iterative utilisation of the first main operation. The test range for plausible $C_{D}$ values [39] is programmatically bounded to the interval $[1-5]$. In this regard, the seed value of $C_{D}$ used during the first propagation is 1 and three successive propagations test remaining values of $C_{D}$ up to 4 . The computed $R_{S M A_{1,2,3}}$ are analysed for interval convergence. If convergence is found, then fine $C_{D}$ fitting is investigated; otherwise the $O P_{t_{N-1} i}$ and $t_{N-1}$ observation are discarded as the orbit propagation sensitivity or the observed data quality is unsupported for analysis. The final fitted drag coefficient $C_{D F_{N-1}}$ is determined by three further iterations using variable $C_{D}$ values defined by their likeliness to minimise $R_{S M A}$. The root-mean square residual on the semi-major axis, $R M S-R_{S M A}$, is computed to report the quality of the estimation. The first and second operations are repeated for all TLE sets available for analysis for each CubeSat.

\footnotetext{
${ }^{3}$ e.g. $\backsim+7.9 \%$ and $\backsim+8.8 \%$ at 280 and $600 \mathrm{~km}$ respectively.

${ }^{4}$ In addition to the JB2008 and GGM05G models, the OP incorporates other important perturbations and considerations like Luni-Solar high-precision ephemeris [23], thermosphere winds (HWM), solar irradiance computed with historical empirical data [35], and conical eclipse conditions [17]
} 


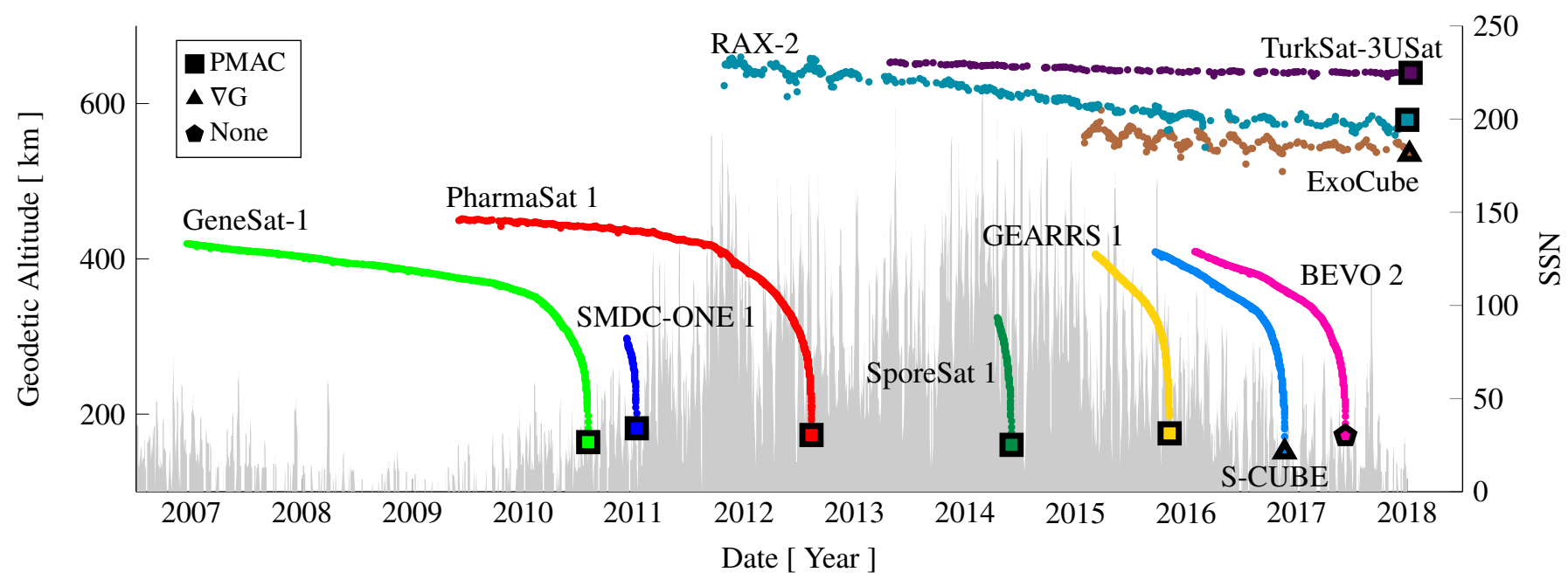

Figure 3: Observed orbital evolution of the analysed CubeSat missions. The sunspot number is used as a proxy for space environment conditions. Plotted data from public sources [12 28].

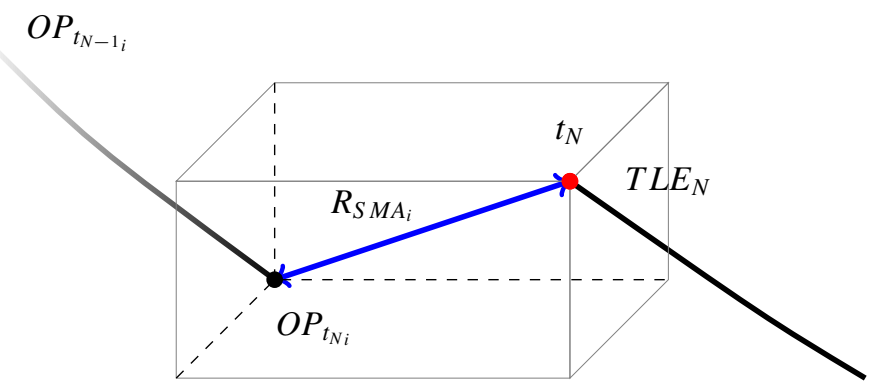

Figure 4: Fitting algorithm. An $i$ number of $C_{D}$ values are used for an equal number of orbital propagations from an initial common observation at time $t_{N-1}$. Each propagation would produce a distinctive value of SMA at $t_{N}$, the time of the successive observation $\left(T L E_{N}\right)$. The iterative process aims to identify a drag coefficient value characterising the interval $t_{N-1}-t_{N}$ by residual minimisation on SMA $\left(R_{S M A_{i}}\right)$ between propagated and observed values.

Note that in this investigation, each reported $R M S-R_{S M A}$ is related to one experiment (one $C_{D F}$ ) and not to a batch of experiments [19]. This approach is adopted because each TLE pair specifies a unique experiment with fundamental characteristics, e.g. unique propagation interval. Although it may be argued that there exists some degree of regularity between TLE sets such as in successive TLE epochs allowing for group statistics of fitted values, in this investigation it is preferred to avoid estimated data softening for each analysed CubeSat.

\subsection{Estimation of Atomic Oxygen Surface Coverage from $C_{D F}$}

The simple three-dimensional computational model assisting the estimation of $A_{d y n}$ values, is also used in the estimation for the computation of the total physical drag coefficient of the CubeSat. In this case, the summation of the individual flat plate drag coefficients for each face are computed with Eq. (8) with their respective values of $B$ (angle of attack). Two extreme total physical values of $C_{D}$ are computed during the fitting process propagations for the given instantaneous environment con- ditions. The first value assumes clean satellite surfaces, $C_{D, s}$, and the second assumes surfaces completely covered by adsorbate in diffuse reflection, $C_{D, a b s}$, i.e. $\sigma_{t}=1$. Whilst in the later case the value of $\alpha_{n}=1$, the clear surface assumption requires the estimation of $\alpha_{n}$. This is achieved using the Goodman's empirical model in a similar approach to Walker et al. [39], Eq. (9], with $\mu$ given by the mass ratio of $\bar{m}_{m}$ and the mass of the surface particles.

$$
\alpha_{n}=\frac{2.4 \mu}{(1+\mu)^{2}}
$$

For each successful identification of $C_{D F}$, the corresponding average values of the two total physical drag coefficients covering that simulation time arc are reported. In this way the values of $\bar{C}_{D, a b s}$ and $\bar{C}_{D, s}$ define a window within which a valid $C_{D F}$ must lie. In order to apply this condition to the final set of $C_{D F}$ for each CubeSat, it is necessary to make an important assumption. Since the $C_{D F}$ values contain unidentified atmospheric bias and other possible sources of error e.g. rounded satellite mass, in this investigation the defined window is displaced to match $\bar{C}_{D, a b s}$ to the limit values of $C_{D F}$ below $250 \mathrm{~km}$. This important assumption is bolstered by the fact that diffuse reflection with complete accommodation is expected to occur within that altitude as discussed earlier. Finally, the estimation of the fraction of surface covered by $\mathrm{AO}$ for each $C_{D F}$ value is made with Eq. 10.

$$
\theta=\frac{C_{D F}-\bar{C}_{D, s}}{\bar{C}_{D, a b s}-\bar{C}_{D, s}}
$$

The estimation of $\alpha_{n}$ for a clear surface requires the estimation of $\mu$ as discussed above, which is dependent upon surface material. In this estimation the atmospheric particles are assumed hard spheres impinging a static lattice (surface). Typical surface materials in CubeSat are varied, e.g. bare metal, coatings, solar cells, etc. In order to assess the repercussion of material selection in the estimation of $\theta$, two assessments in GeneSat-1 were carried out. One of the analyses assumes aluminium surfaces ( $27 \mathrm{amu}$ ) and the other assumes glass surfaces 
(60 amu [15], typically found as solar cell protection). Results of both studies are reported in Fig. 5 . From the figure is observed that the fraction of glass surface covered by $\mathrm{AO}$ is biased $\sim+15 \%$ with respect to the aluminium surface, and that the level of uncertainty increases at lower $\theta$. Considering that the glass surface percentage is highly variable amongst CubeSat missions, the following analyses are computed assuming aluminium surfaces sticking to the CubeSat Design Specification 5 .

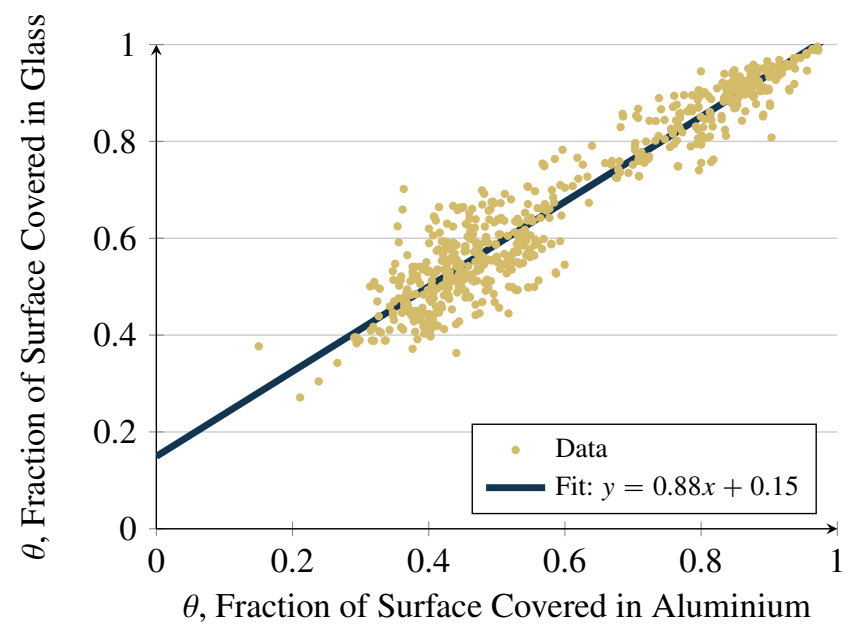

Figure 5: Estimated fraction of surface covered by AO for the cases of aluminium and glass in GeneSat-1.

\subsection{Analysis of Results}

The performance of the fitting process reported in this investigation is presented in Table 3 In all instances the inherent accumulation of uncertainties of the TLE-OP-TLE analysis, viz. fitting process, is indicated in the low success percentages in Table 3 The best drag coefficient fitting performance is reported for SporeSat 1. On the other hand, the least performance is reported for TurkSat-3USat. Since these cases correspond to extreme atmospheric conditions, that is high solar activity at low orbital altitude and prevalently low solar activity at high orbital altitude (Fig. 3), the extreme performance results in Table 3 verify that the fitted values are related to atmospheric drag. Whenever the drag force is weak and overwhelmed by computational/observational uncertainties the success percentage is low.

Table 5 summarises the main results of the $C_{D F}$ studies. The table reports the box-and-whisker plots for $C_{D F}$, the root-mean square residuals $R M S-R_{S M A}$, the average dynamic cross sectional areas $\bar{A}_{d y n}$, and the average angle of incidence of atmospheric species $\bar{\phi}_{i c}$. From Fig. 3 we see that seven orbits terminated with reentry, and the remaining three continue with their natural orbital decay by January 2018. Terminated orbits show

${ }^{5}$ Point 3.2.15 in the CDS Rev. 13 [4] states that Aluminium 7075, 6061, 5005, and/or 5052 will be used for both the main CubeSat structure and the rails.
Table 3: $C_{D F}$ fitting process success percentage.

\begin{tabular}{lccr}
\hline Name & TLE sets & Number of $C_{D F}$ & Success [ \% ] \\
\hline BEVO 2 & 821 & 401 & 48.84 \\
ExoCube & 2825 & 209 & 7.40 \\
GEARRS 1 & 588 & 420 & 71.43 \\
GeneSat-1 & 2164 & 803 & 37.11 \\
PharmaSat 1 & 1894 & 769 & 40.60 \\
RAX-2 & 3036 & 443 & 14.59 \\
SMDC-ONE 1 & 83 & 65 & 78.31 \\
SporeSat 1 & 125 & 112 & 89.60 \\
S-CUBE & 816 & 553 & 67.77 \\
TurkSat-3USat & 4751 & 171 & 3.60 \\
\hline
\end{tabular}

contained $C_{D F}$ values below three, whereas those for ExoCube, TurkSat-3USat, and RAX-2 show higher values with maximum of $\bar{C}_{D F}$ of 4.43 reported for TurkSat-3USat. The $C_{D F}$ results are congruent with the solar activity levels experienced by the CubeSat along their respective orbital evolution. For instance, $C_{D F}$ results for SporeSat 1 show reduced data dispersion centred in a low value $\left(\bar{C}_{D F}=2.03\right)$, consistent with its orbital altitude (near-circular orbit below $320 \mathrm{~km}$ at inclination $51.6^{\circ}$ as shown in Fig. 6) and high solar activity. In another example, the ExoCube orbiting at $400-670 \mathrm{~km}$ in polar orbit during mostly low solar activity, reports dispersed fitted values centred at $\bar{C}_{D F}=3.20$.

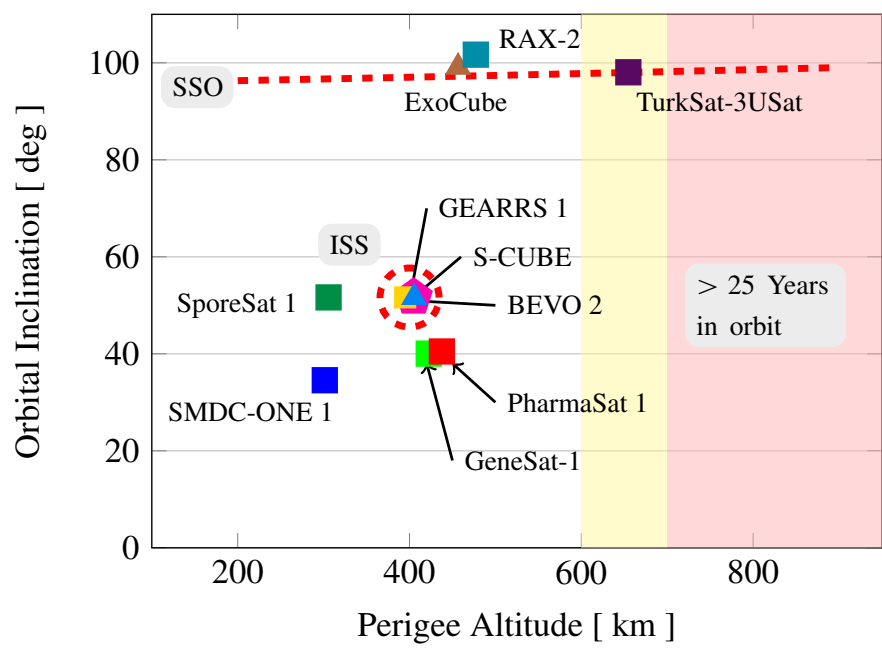

Figure 6: Distribution in the perigee altitude-inclination space of the analysed CubeSats. Sun-synchronous orbits (SSO) and satellites deployed from the International Space Station (ISS) are shown. Orbit lifespan ranges from de Selding [8].

$R M S-R_{S M A}$ values show high dispersion in orbits subject to high atmospheric drag. The reason for this is attributed to a difference between the $C_{D F}$ and the true drag coefficient value representing the time arc under simulation. Although small, such a difference could produce high residuals during strong atmospheric drag episodes. For this reason, the reentered SMDCONE 1 and SporeSat 1 that orbited during moderate-to-high solar activity, report the highest values and $R M S-R_{S M A}$ disper- 
sion. Conversely, the least values of $R M S-R_{S M A}$ are expected in ExoCube, TurkSat-3USat, and RAX-2.

Finally, dynamic cross-sectional areas and average bulk atmosphere angles of incidence are reported in Table 5. In this investigation, the use of $A_{d y n}$ is assumed a fundamental improvement in the estimation of $C_{D F}$. In order to assess the validity of this assumption, results from this study are compared with an external investigation using fixed cross-sectional areas. Fitted drag coefficient values reported by Oltrogge and Leveque [18] were estimated using fixed mean cross-sectional areas of $350 \mathrm{~cm}^{2}$ indistinctly in concave and convex $3 \mathrm{U}$ CubeSats. Their study encompasses the orbital decay of various CubeSats up to the year 2011. Since in our investigation the attention is centred on convex geometries, the group of coincident missions is reduced to SMDC-ONE 1, GeneSat-1, and PharmaSat 1. In order to perform adequate comparison of these results to those reported in this investigation it is necessary to make some assumptions. Firstly, the reported $\bar{C}_{D F}$ values in the study of Oltrogge and Leveque [18] were computed with four different atmospheric density models, i.e. the Jacchia-Bowman Thermospheric density model 2006 (JB2006), Jacchia 1971, the Standard Atmosphere 1976, and the MSISE2000. Results from the JB2006 are preferred for the purpose of comparison in this investigation due to atmospheric model similarities ${ }^{6}$ Secondly, as the exact date of the analysed time intervals are not provided, it is assumed that their analysis is delimited by June 2011, the date of presentation of the work. This assumption has no effect on the comparison of SMDC-ONE 1 and GeneSat- $1 \bar{C}_{D F}$ values because both orbits terminated in early 2011 as shown in Fig. 3. On the other hand only $\bar{C}_{D F}$ values before June 2011 from this investigation are used in the case of PharmaSat 1.

Table 4 presents the $\bar{C}_{D F}$ values from both investigations for the missions in common. The first aspect to point out is that some level of correction for atmospheric density bias is required in both $\bar{C}_{D F}$ investigations 7 , as suggested by the values below 2.0 in Table 4, This owing to the unattainability of such values in free molecular flow as verified from Eq. (4) for $B=90^{\circ}$ wherein $\lim _{\left(\alpha_{n}, s\right) \rightarrow(1, \infty)} C_{D}\left(\alpha_{n}, s\right)=2$ (hyperthermal free molecular flow condition), or from Walker et al. [39]. Whilst discrepancies in $\bar{C}_{D F}$ values for GeneSat-1 may be explained to some extent to different biases of atmospheric model, those of PharmaSat 1 suggest fundamental differences between the methods of estimation. From Fig. 3 is observed that the time range under analysis for PharmaSat 1 is characterised by orbital altitudes encompassing $420-450 \mathrm{~km}$ and low-to-moderate solar activity. Under these conditions the value of $C_{D}$ is expected to be relatively high. As a reference for comparison, note that the values for GeneSat-1 in Table 4 cover a period of low solar activity. Additionally, during the portion of rapid orbital decay low values of $C_{D F}$ contribute to the reduction of the reported average. In a rough comparison, $\bar{C}_{D F}$ values for PharmaSat 1

\footnotetext{
${ }^{6}$ Both, the JB2006 and JB2008 models are based on total density estimations from satellite drag [36]. In addition the JB2006 already comprises the improved formulation for high energy incoming solar radiation used in the JB2008 [3]

${ }^{7}$ Bias in JB2006 was identified by Pardini et al. [20] from $+11.6 \%$ to $+15.1 \%$ at $272 \mathrm{~km}$ and $476 \mathrm{~km}$ respectively during the $23^{r d}$ sunspot maximum
}

should be higher or at least similar to those for GeneSat-1. On the other hand, values for SMDC-ONE 1 are expected to be low due to low orbital altitude during moderate solar activity. The representativeness of the $\bar{C}_{D F}$ values from this investigation, are attributed to the implementation of $A_{d y n}$ as different bias in the atmospheric models can hardly explain discrepancies.

Table 4: Comparison of estimated $\bar{C}_{D F}$ values from this investigation and those reported by Oltrogge and Leveque. Standard deviation reported in parentheses.

\begin{tabular}{lccr}
\hline Name & $\begin{array}{c}\text { This } \\
\text { investigation }\end{array}$ & $\begin{array}{c}\text { Oltrogge } \\
\text { and Leveque }\end{array}$ & $\begin{array}{r}\text { Rel. Error } \\
\text { \% }\end{array}$ \\
\hline GeneSat-1 & $2.38(0.28)$ & 2.85 & 19.75 \\
PharmaSat 1 & $2.79(0.39)$ & 1.35 & -51.61 \\
SMDC-ONE 1 & $1.96(0.13)$ & 2.54 & 29.59 \\
\hline
\end{tabular}

${ }^{\star}$ With respect to the values of this investigation

The ultimate objective of this investigation is the estimation of AO surface coverage from the $C_{D F}$. In this regard, the simple Langmuir adsorption model in Eq. 11 has been used in larger class satellites to provide a theoretical framework to the observed fraction of the surface covered by $\mathrm{AO}$ as function of its partial pressure. This model assumes that the AO behaves like an ideal gas at isothermal conditions, which under the influence of the partial pressure produces monolayer adsorption on the surface. Although this assumption may be an oversimplification under specific atmospheric circumstances. For instance, Pilinski et al. [21] suggested that AO secondary adsorption or recombination may occur in explanation to values lying below the the Langmuir adsorption model.

$$
\theta=\frac{K P_{A O}}{1+K P_{A O}}
$$

In spite of generalisation limitations of the Langmuir adsorption model, it may provide physical underpinning and validation of $\theta$ values derived from $C_{D F}$. To this objective, the model could be statistically fitted to the estimations through the adsorbate constant $K$. Values of $\theta$ estimated with the $C_{D}$ window described in subsection 2.1. have been divided into two groups according to the orbital inclination of the missions for illustration purposes. Note that no estimations of $\theta$ were possible for TurkSat-3USat, RAX-2, and ExoCube, due to the lack of information to adequately fix the $C_{D}$ window (using $\bar{C}_{D, a b s}$ ). Fig. 7 and Fig. 8 show the estimated data along with the Langmuir adsorption model fit of the major contributor of estimations. In Fig. 7 the fitted value of $K$ corresponds to values from PharmaSat 1, and in Fig. 8 corresponds to S-CUBE. The best fit of the adsorbate constant for PharmaSat 1 is $K_{P h}=1.273 E+06$, and for SCUBE is $K_{S C}=6.586 E+05$. These values show agreement to the values of $K$ reported by Walker et al. [39] and Pilinski et al. [21] obtained from larger class satellites (from $K=1.4 E+03$ to $2.9 E+06$ form various GSI models, and $K=5.4 E+06$ respectively.).

In Fig. 7 is observed that whilst some data is well represented by a Langmuir fit, there may be important deviations from it. Detailed investigation of the possible causes of abnormal trends 
will be the subject of future work. However, sustained increment of solar activity and strong solar flares are identified as probable drivers. For instance, in PharmaSat 1 is presented a region of $\theta$ values lower than the Langmuir fit. Some of these values coincide with the occurrence of a strong solar flare (M7.9) and geomagnetic storm (G2 moderate) on 13 March 2012 [31]. In GeneSat-1 a group of values is systematically higher than a Langmuir fit. These values are related to the sustained increment in solar observed during the end of the year 2009 and beginning of 2010 as shown in Fig. 3 .

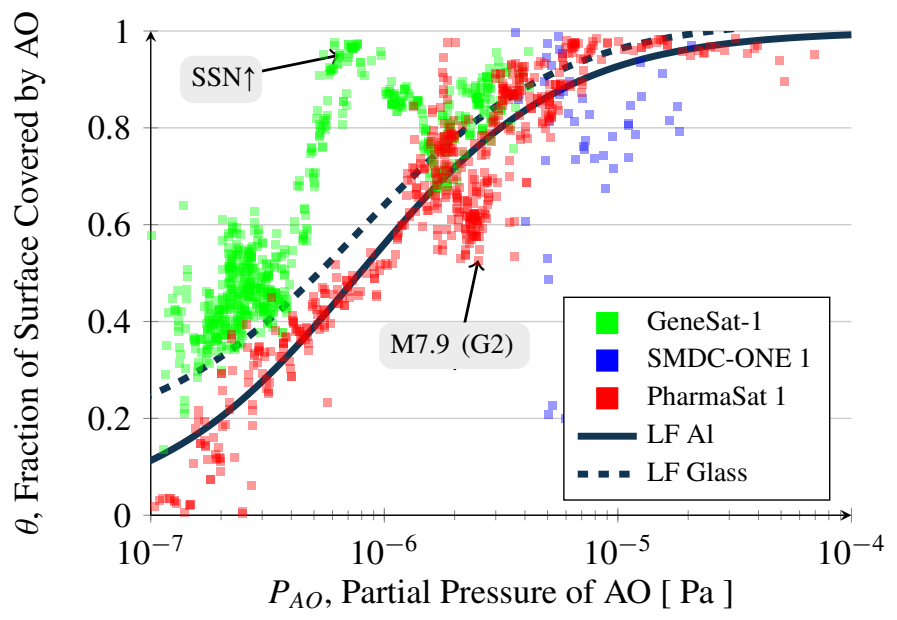

Figure 7: Group of CubeSats at orbital inclinations of approximately $40^{\circ}$. Labels identify data related to space environment events. The solid line shows the Langmuir fit (LF) for an aluminium surface, and the dashed line shows that of a glass surface to indicate possible variation due to material uncertainty.

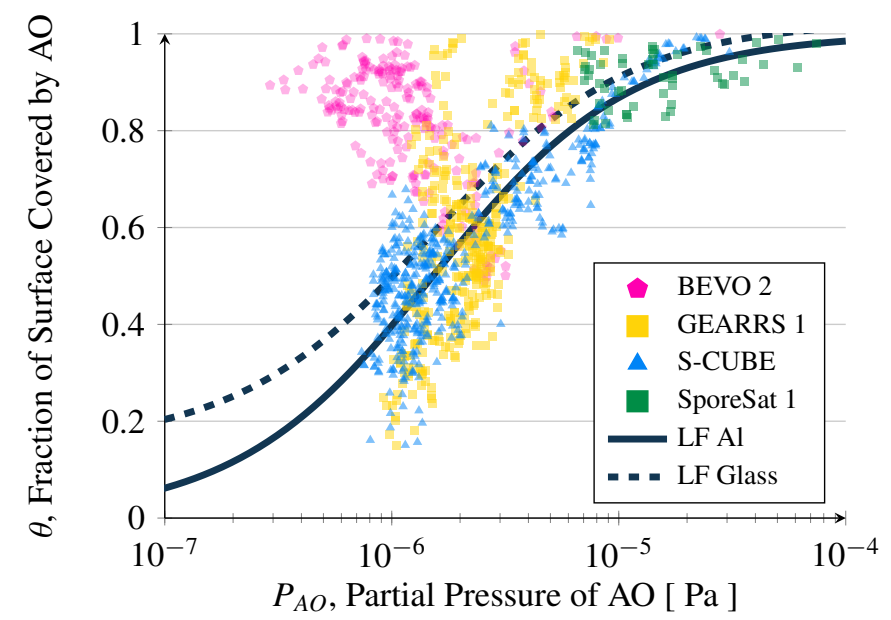

Figure 8: Same as Fig. 7 for CubeSats at orbital inclinations of approximately $50^{\circ}$.

Interestingly, a Langmuir fit would be unrepresentative for the $\theta$ values of BEVO 2 in Fig. 8. Aiming at the clarification of this issue, further study of BEVO 2 was carried out. The possibility that BEVO 2 followed an unintended $\nabla G$ profile instead of random tumbling motivated a new study. Fitting results show that the $\nabla G$ profile is more representative of BEVO 2 by incrementing the fitting success by $41.3 \%$. Notwithstanding this improvement, $\theta$ values showed the same abnormal trend in the $\theta$-Pressure space although displaced $1.5 \times 10^{-6} \mathrm{~Pa}$ to the left. The reason of this abnormal trend remains unclear. Finally, the consolidation of $\theta$ results as function of altitude is shown in Fig. 9. The Langmuir curves for extreme solar activity conditions enclose most data.

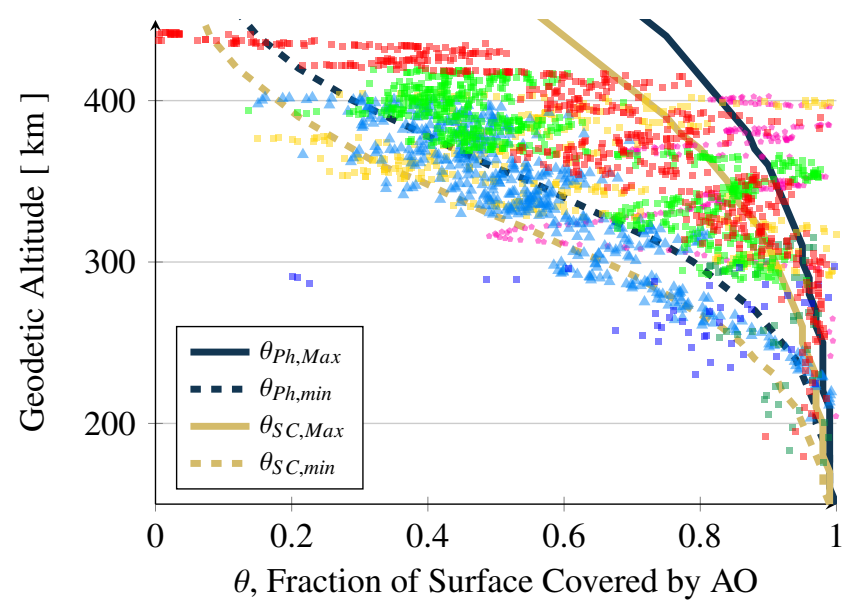

Figure 9: Consolidation of fractional surface coverage as function of orbital altitude. The curves show the Langmuir adsorption model $\left(K_{P h}\right.$ and $\left.K_{S C}\right)$ trends of aluminium for maximum and minimum solar activity conditions.

\section{Conclusions}

This investigation set out to determine distinctive drag coefficient development and attributes of atmospheric gas-surface interactions in nanosatellites. The selected $3 \mathrm{U}$ CubeSats for this investigation, BEVO 2, ExoCube, GEARRS 1, GeneSat1, PharmaSat 1, RAX-2, SMDC-ONE 1, SporeSat 1, S-CUBE, and TurkSat-3USat, use passive attitude stabilisation strategies and have strong convex geometry attributes. Dynamic crosssectional areas were employed in the fitting of drag coefficients instead of the customary use of constant values targeting a better description of the time-varying projected geometry of the standard 3U CubeSat, and therefore representativeness of the results. Estimations of atomic oxygen surface coverage were estimated by means of a drag coefficient closed form solution. The quality of the atomic oxygen surface coverage results was tested using the Langmuir adsorption model.

Based upon the evidence collected in the investigation and the comparison to external data, the fitted drag coefficients computed with dynamic cross-sectional areas provide consistent and descriptive data for the geometry and orbital characteristics of the analysed CubeSats. Additionally, results show that estimating atomic oxygen surface coverage using fitted drag values is viable. 


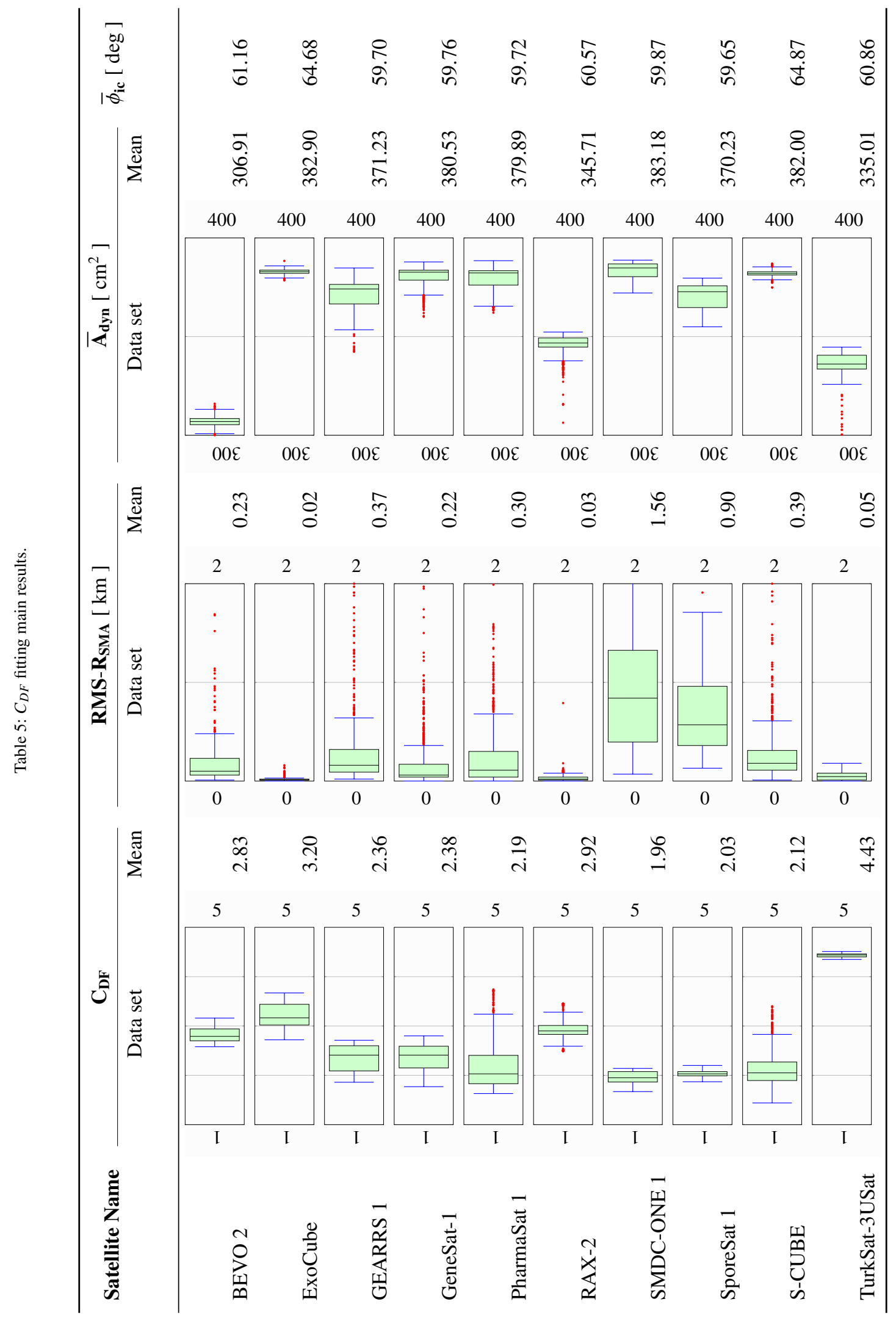




\section{References}

[1] Bettadpur, S., Ries, J., Eanes, R., Nagel, P., Pie, N., Poole, S., Richter, T., and Save, H. (2015). Evaluation of the GGM05 Mean Earth Gravity Model. In EGU General Assembly Conference Abstracts, volume 17 of EGU General Assembly Conference Abstracts, page 4153.

[2] BGS and NCEI (2017). World magnetic model.

[3] Bowman, B. R., Tobiska, W. K., Marcos, F. A., Huang, C. Y., Lin, C. S., and Burke, W. J. (2008). A new empirical thermospheric density model JB2008 using new solar and geomagnetic indices. In AIAA/AAS astrodynamics specialist conference and exhibit, Honolulu, Hawaii.

[4] Cal Poly (2015). CubeSat Design Specification (CDS) Rev 13. Cal Poly SLO, 2015 edition.

[5] Cercignani, C. and Lampis, M. (1971). Kinetic models for gas-surface interactions. transport theory and statistical physics, 1(2):101-114.

[6] Cook, G. (1965). Satellite drag coefficients. Planetary and Space Science, 13(10):929-946.

[7] De Groh, K. K. and McCue, T. R. (1999). Analyses of contaminated solar array handrail samples retrieved from Mir. Technical report, SAE Technical Paper.

[8] de Selding, P. B. (2018). 1 in 5 CubeSats violates international orbit disposal guidelines.

[9] ECSS (2008). Ecss space engineering-space environment. Technical report, Technical Report ECSS-E-ST-10-04C, ESA.

[10] Fahnestock, E. G., Park, R. S., Yuan, D.-N., and Konopliv, A. S. (2012). Spacecraft thermal and optical modeling impacts on estimation of the GRAIL lunar gravity field. In AIAA/AAS Astrodynamics Specialist Conference, pages 13-16.

[11] Izakov, M. (1965). Some problems of investigating the structure of the upper atmosphere and constructing its models. Space Research, 5:1191.

[12] Kelso, T. S. (2017). Satellite catalog (satcat).

[13] Li, A. and Close, S. (2015). Mean thermospheric density estimation derived from satellite constellations. Advances in Space Research, 56(8):1645-1657.

[14] Moe, K. and Bowman, B. (2005). The effects of surface composition and treatment on drag coefficients of spherical satellites, AAS 2005-258. American Astronautical Society Publications Office, San Diego, CA.

[15] Moe, K. and Moe, M. M. (2005). Gas-surface interactions and satellite drag coefficients. Planetary and Space Science, 53(8):793-801.

[16] Moe, K., Moe, M. M., Levin, D. A., Wysong, I. J., and Garcia, A. L. (2011). Gas-surface interactions in Low-Earth Orbit. In AIP Conference Proceedings, volume 1333, pages 1313-1318. AIP.

[17] Neta, B. and Vallado, D. (1997). On satellite umbra/penumbra entry and exit positions. Technical report, Monterey, California. Naval Postgraduate School.

[18] Oltrogge, D. and Leveque, K. (USA, 2011). An evaluation of CubeSat orbital decay. In AIAA/USU Conference on Small Satellites, volume SSC11VII-2 AIAA/USU.

[19] Pardini, C., Anselmo, L., Moe, K., and Moe, M. (2010). Drag and energy accommodation coefficients during sunspot maximum. Advances in Space Research, 45(5):638-650.

[20] Pardini, C., Moe, K., and Anselmo, L. (2012). Thermospheric density model biases at the 23rd sunspot maximum. Planetary and Space Science, 67(1): $130-146$

[21] Pilinski, M. D., Argrow, B. M., and Palo, S. E. (2010). Semiempirical model for satellite energy-accommodation coefficients. Journal of Spacecraft and Rockets, 47(6):951-956.

[22] Pilinski, M. D., Argrow, B. M., and Palo, S. E. (2011). Drag coefficients of satellites with concave geometries: comparing models and observations. Journal of Spacecraft and Rockets, 48(2):312-325.

[23] Reda, I. (2010). Solar eclipse monitoring for solar energy applications using the solar and moon position algorithms. Publications $(E)$.

[24] Saunders, A., Swinerd, G. G., and Lewis, H. G. (2012). Deriving accurate satellite ballistic coefficients from two-line element data. Journal of Spacecraft and Rockets, 49(1):175-184.

[25] Sentman, L. H. (1961). Free molecule flow theory and its application to the determination of aerodynamic forces. Technical report, DTIC Document.

[26] Shen, C. (2006). Rarefied gas dynamics: fundamentals, simulations and micro flows. Springer Science \& Business Media.

[27] Shim, J., Kuznetsova, M., Rastätter, L., Hesse, M., Bilitza, D., Butala, M., Codrescu, M., Emery, B., Foster, B., Fuller-Rowell, T., et al. (2011).
CEDAR electrodynamics thermosphere ionosphere (ETI) challenge for systematic assessment of ionosphere/thermosphere models: NmF2, hmF2, and vertical drift using ground-based observations. Space Weather, 9(12).

[28] SILSO (2017). Sunspot number.

[29] Space Environment Technologies (2017). The Jacchia-Bowman 2008 Empirical Thermospheric Density Model.

[30] Space-Track (2017). Two Line Element (TLE) Data.

[31] SpaceWeatherLive (2018). Top 50 solar flares of the year 2012.

[32] Springmann, J., Kempke, B., Cutler, J., and Bahcivan, H. (2012). Initial flight results of the RAX-2 satellite. 26th Annual AIAA/USU Conference on Small Satellites.

[33] Storch, J. (2002). Aerodynamic disturbances on spacecraft in freemolecular flow. Technical report, DTIC Document.

[34] The CIRA Working Group (2012). Cospar international reference atmosphere - 2012. Technical report, The Committee on Space research.

[35] University of Colorado (2017). SORCE SOlar Radiation and Climate Experiment.

[36] Vallado, D. A. (2013). Fundamentals of Astrodynamics and Applications. Space Technology Library.

[37] Vallado, D. A. and McClain, W. D. (2001). Fundamentals of astrodynamics and applications, volume 12. Springer Science \& Business Media.

[38] Vaughan, W. (2010). Guide to reference and standard atmosphere models. Technical report, AIAA-G-003C-2010, American Institute of Aeronautics and Astronautics, Reston, VA, from http://www. aiaa. org.

[39] Walker, A., Mehta, P., and Koller, J. (2014). Drag Coefficient Model Using the Cercignani-Lampis-Lord Gas-Surface Interaction Model. Journal of Spacecraft and Rockets, 51(5):1544-1563.

[40] Wolverton, R. W. (1961). Flight performance handbook for orbital operations. John Wiley and Sons. 